\title{
Impairment of right ventricular longitudinal strain associated with severity of pneumonia in patients recovered from COVID-19
}

\author{
Pelin Karaca Ozer ${ }^{1}$ ( ) Elif Ayduk Govdeli ${ }^{1}$ - Derya Baykiz ${ }^{1}$. Ekrem Bilal Karaayvaz ${ }^{1}$ Alpay Medetalibeyoglu ${ }^{2}$. \\ Yunus Catma ${ }^{2} \cdot$ Ali Elitok $^{1} \cdot$ Atahan Cagatay $^{3} \cdot$ Berrin Umman $^{1} \cdot$ Aytac Oncul $^{1} \cdot$ Tufan Tukek $^{2} \cdot$ Zehra Bugra $^{1}$
}

Received: 20 January 2021 / Accepted: 1 March 2021 / Published online: 11 April 2021

(c) The Author(s), under exclusive licence to Springer Nature B.V. 2021

\begin{abstract}
Myocardial injury caused by COVID-19 was reported in hospitalized patients previously. But the information about cardiac consequences of COVID-19 after recovery is limited. The aim of the study was comprehensive echocardiography assessment of right ventricular (RV) in patients recovered from COVID-19. This is a prospective, single-center study. After recovery from COVID-19, echocardiography was performed in consecutive 79 patients that attended follow-up visits from July 15 to November 30, 2020. According to the recovery at home vs hospital, patients were divided into two groups: home recovery $(n=43)$ and hospital recovery $(n=36)$. Comparisons were made with age, sex and risk factor-matched control group $(n=41)$. In addition to conventional echocardiography parameters, RV global longitudinal strain (RV-GLS) and RV free wall strain (RV-FWS) were determined using 2D speckle-tracking echocardiography (2D STE). Of the 79 patients recovered from COVID-19, 43 (55\%) recovered at home, while 36 (45\%) required hospitalization. The median follow-up duration was $133 \pm 35$ (87-184) days. In patients recovered from hospital, RV-GLS and RV-FWS were impaired compared to control group (RV-GLS: $-17.3 \pm 6.8$ vs. $-20.4 \pm 4.9$, respectively [p=0.042]; RV-FWS: $-19.0 \pm 8.2$ vs. $-23.4 \pm 6.2$, respectively $[\mathrm{p}=0.022])$. In subgroup analysis, RV-FWS was impaired in patients severe pneumonia $(\mathrm{n}=11)$ compared to mild-moderate pneumonia $(\mathrm{n}=28)$, without pneumonia $(\mathrm{n}=40)$ and control groups $(-15.8 \pm 7.6$ vs. $-21.6 \pm 7.6$ vs. $-20.8 \pm 7.7$ vs. $-23.4 \pm 6.2$, respectively, $[\mathrm{p}=0.001$ for each]) and RV-GLS was impaired compared to control group $(-15.2 \pm 6.9$ vs. $-20.4 \pm 4$; respectively, $[\mathrm{p}=0.013])$. A significant correlation was detected between serum CRP level at hospital admission and both RV-GLS and RV-FWS $(r=0.285, p=0.006 ; r=0.294, p=0.004$, respectively). Age (OR 0.948, $\mathrm{p}=0.010)$, male gender (OR 0.289, $\mathrm{p}=0.009)$, pneumonia on CT (OR 0.019, $\mathrm{p}=0.004)$, and need of steroid in treatment (OR 17.424, $\mathrm{p}=0.038$ ) were identifed as independent predictors of impaired RV-FWS $(>-18)$ via multivariate analysis. We demonstrated subclinic dysfunction of RV by 2D-STE in hospitalized patients in relation to the severity of pneumonia after recovery from COVID-19. 2D-STE supplies additional information above standard measures of RV in this cohort and can be used in the follow-up of these patients.
\end{abstract}

Keywords COVID-19 $\cdot$ Pneumonia $\cdot$ Recovery $\cdot$ Right ventricle $\cdot$ Speckle tracking echocardiography

\section{Introduction}

Pelin Karaca Ozer

pkaracaozer@gmail.com

1 Istanbul Medical Faculty, Department of Cardiology, Istanbul University, Topkapi Mahallesi, Turgut Ozal Millet Caddesi, 34093 Fatih/Istanbul, Turkey

2 Istanbul Medical Faculty, Department of Internal Medicine, Istanbul University, Istanbul, Turkey

3 Istanbul Medical Faculty, Department of Infectious Diseases and Clinical Microbiology, Istanbul University, Istanbul, Turkey
The coronavirus disease 2019 (COVID-19) caused by severe acute respiratory syndrome coronavirus 2 (SARS-CoV-2) involves primarily the respiratory system, but also can lead to cardiac impairment $[1,2]$. Currently studies have demonstrated the presence of myocardial damage, including left ventricular (LV) dysfunction and myocarditis, associated with in-hospital mortality in COVID-19 patients [2-4]. The lungs are the major organ involved in COVID-19, so right ventricle (RV) is more likely to be affected compared to LV because of increased RV afterload. Previous studies 
reported RV dysfunction in patients with acute respiratory distress syndrome and respiratory failure [5, 6]. But limited information is available for RV involvement in COVID-19 patients, especially after recovery. Standard measurements of conventional echocardiography may be insufficient to detect subclinical abnormalities of RV [5, 7]. It has been demonstrated that RV longitudinal strain (RVLS) obtained from two-dimensional speckle tracking echocardiography (2D STE) is an objective and sensitive method for detecting silent impairment of RV systolic function [7, 8]. So far, RVLS has been studied and shown to be associated with prognosis in hospitalized patients with COVID-19, defined as a better predictor of mortality than conventional parameters of RV $[9,10]$. To the best of our knowledge, assessment of RV with 2D STE has not been reported in patients recovered from COVID-19.

\section{Materials and methods}

\section{Study design and population}

After recovery, echocardiography was performed in consecutive 79 patients that attended follow-up visits at Istanbul University Faculty of Medicine COVID-19 Outpatient Clinic from July 15 to November 30, 2020.

All other clinical conditions that may impair RV strain analysis were determined as the exclusion criteria. In this respect, patients with older age ( $>85$ years old), preexisting cardiovascular disease (heart failure, coronary artery disease, valvular heart disease), atrial fibrillation, $\geq$ stage 2 hypertension (HTN), uncontrolled diabetes mellitus (DM) (HbA1c $\geq 8$ ), prior cerebrovascular disease, chronic liver or kidney disease (GFR $<30 \mathrm{ml} / \mathrm{min}$ ), asthma, chronic obstructive pulmonary disease, prior history of pulmonary hypertension and pulmonary embolism, malignancy and poor echogenicity were excluded from the study. Comparisons were made with age, sex and risk factor-matched control group $(n=41)$ that had no history of a respiratory infection. Two dimensional transthoracic echocardiography (2D TTE) and 2D STE was performed in all subject.

According to the recovery at home vs hospital, COVID19 patients were divided into two groups: home recovery and hospital recovery. For subgroup analysis, COVID-19 patients were divided into three groups according to the severity of pneumonia or the absence of pneumonia: without pneumonia, mild-moderate pneumonia, severe pneumonia.

The study was registered in the Ministry of Health COVID-19 research registry and approved by the Istanbul Faculty of Medicine Ethics Board (Clinical trial registration number: 2020/1185, approval number: 1303). All patients gave informed consent.

\section{Data collection}

Clinical demographic characteristics, echocardiography measurements and blood samples of patients were obtained at follow-up visits. Again blood samples were obtained from control group on the day of echocardiography for comparison with patients.

Laboratory results included complete blood cell analysis, C-reactive protein (CRP), ferritin, high-sensitivity troponin $\mathrm{T}$ (hs-TnT), pro-BNP and D-dimer for all subject.

The laboratory parameters of patients at hospital admission and medications for COVID-19 were retrospectively collected from medical records. The images from thorax computed tomography (CT) scans which were obtained at hospital admission were retrieved from the picture archiving and communication system (PACS) for all COVID-19 patients.

\section{Two-dimensional transthoracic echocardiography}

Echocardiography was performed with use of appropriate personal protective equipment by investigators who were blinded to the clinical and laboratory data. The date of COVID-19 diagnosis was considered as the index date to calculate the timing of cardiac ultrasound that was performed after a median of $133 \pm 35$ (87-184) days. Examinations were performed using the Vivid 7 echocardiography device (General Electrics, Milwaukee,WI) using a middlerange frequency $(3-8 \mathrm{MHz}$ ) broadband transducer to evaluate parasternal and apical images (2D, M-mode, Doppler echocardiography), with the patient placed in the left lateral decubitus position. Echocardiographic images were obtained using the techniques recommended by the American Society of Echocardiography (ASE) guidelines [11].

\section{Conventional echocardiographic analysis}

Structure of cardiac chambers and left and right ventricular systolic and diastolic function were assessed. LV end-systolic volume (LVESV), LV end-diastolic volume (LVEDV) and LV ejection fraction (LVEF) were measured using the biplane Simpson method. LV mass was calculated on the basis of Devereux's formula. LV diastolic function was estimated using the ratio of early transmitral flow velocity (E) to late transmitral flow velocity (A) and the ratio of transmitral E to early diastolic medial septal tissue velocity (e'). Left atrial volume was calculated using the biplane method in four- and two-chamber views, and it was indexed to BSA for left atrial volume index (LAVI). Right atrial (RA) and RV size were determined from the apical 4-chamber view. Tricuspid annular plane systolic excursion (TAPSE) was 
measured as the systolic displacement of the tricuspid lateral annulus on M-mode imaging. RV fractional area change (RVFAC) was calculated as (RV end-diastolic area RV endsystolic area)/end-diastolic area 100\%. Tricuspid lateral annular systolic velocity (TDI S') was assessed using tissue Doppler imaging from the apical 4- chamber view. Pulmonary artery systolic pressure (sPAP) was assessed from the peak velocity of the tricuspid regurgitation jet, using the modified Bernoulli equation plus right atrial pressure evaluated from the inferior vena cava size and its collapsibility [12].

\section{Speckle-tracking echocardiographic analysis}

2D STE was utilised to characterise longitudinal systolic strain according to the recommendations of the American Society of Echocardiography and the European Association of Cardiovascular Imaging [13]. Images were acquired at 70-100 frames per second at end-expiration in the four (4C)-chamber view and analysed in a blinded manner, offline using a dedicated software package (Automatic Function Imaging (AFI), EchoPac.PC; GE Healthcare, USA). Using the AFI, a point-and-click approach was utilised to identify three anchor points (two basal and one apical), following which the software tracked the endocardial border of the RV automatically. Manual adjustment was performed to ensure adequate tracking. The RV was divided into 6 segments for analysis (basal free wall, mid-free wall, apical free wall, basal septum, mid septum, and apical septum). Longitudinal strain (LS) was obtained by comparing displacement of the speckles relative to each other throughout the cardiac cycle for each segment. RVLS parameters assessed included average RV free wall strain (RV-FWS), and RV global longitudinal strain (RV-GLS) [14, 15]. RV-GLS was defined as the average of all $6 \mathrm{RV}$ segments. RV-FWS was calculated as the mean of the strain values in the three segments of the RV free wall (Fig. 1). Segments that could not be tracked after manual adjustment by the operator were excluded.

\section{Definitions}

According to the World Health Organization (WHO) guidance, the diagnosis of COVID-19 is based on a real-time reverse-transcription polymerase chain reaction (RT-PCR) test. SARS-CoV-2 RNA was detected using the real-time RT-PCR method in the Public Health Microbiology Reference Laboratory of the Ministry of Health. COVID-19 patients were considered as recovered when they discharged from hospital or if they had resolution of symptoms and negative results on a swab test at the end of the isolation period at home.

The severe pneumonia was defined with any of the following in patients with laboratory and thorax CT-confirmed interstitial pneumonia: (1) respiratory distress (respiratory rate $\geq 30$ breaths per min); (2) oxygen saturation at rest $\leq 93 \%$; (3) ratio of the partial pressure of arterial oxygen $(\mathrm{PaO} 2)$ to the fractional concentration of oxygen inspired air (FiO2) $(\mathrm{PaO} 2: \mathrm{FiO} 2, \leq 300 \mathrm{mmHg})$; or (4) a critical complication (respiratory failure and mechanical ventilation required, septic shock, and/or multiple organ dysfunction/ failure and intensive care unit admission required) [16].

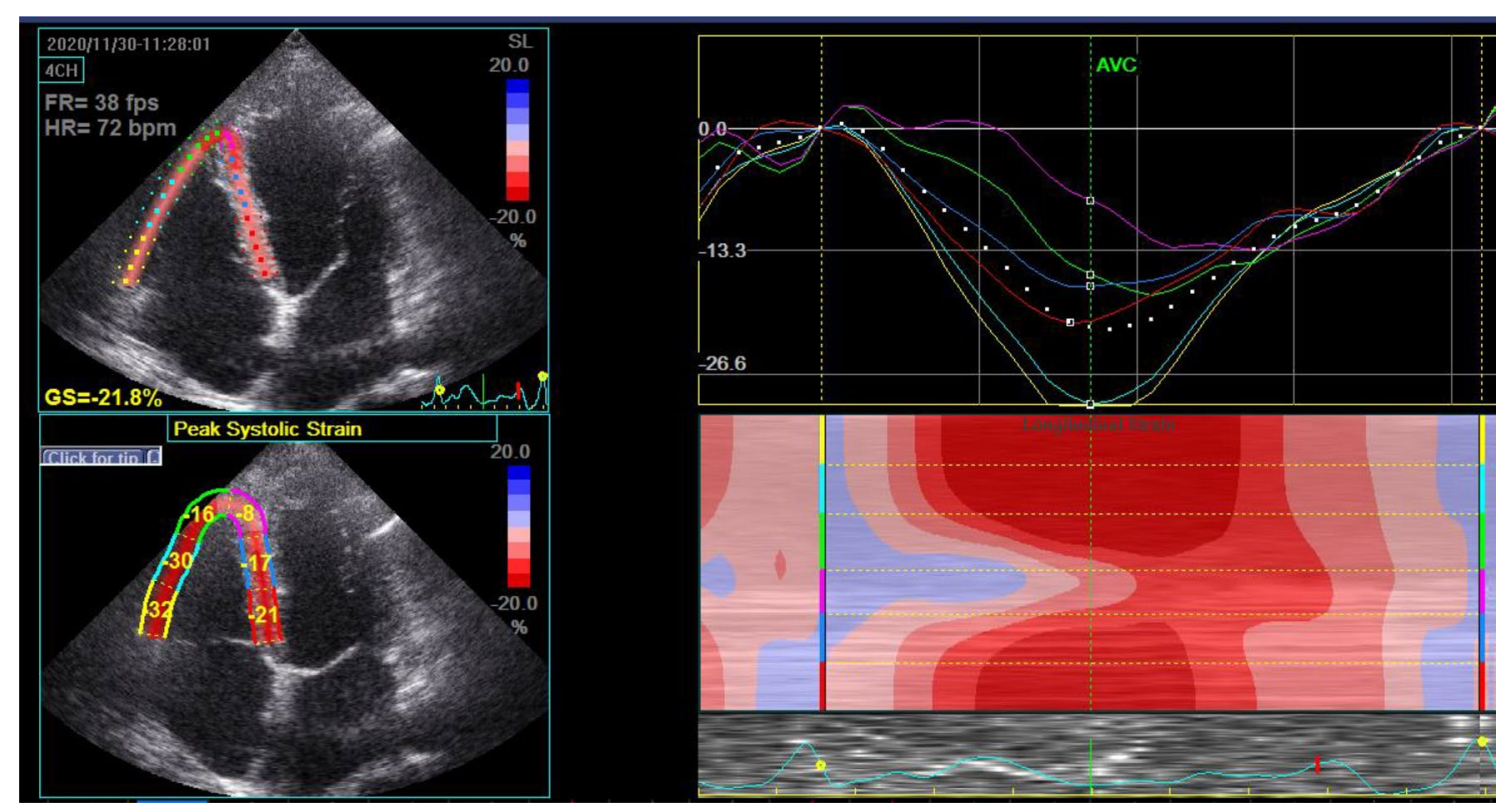

Fig. 1 Apical four-chamber view of RV longitudinal strain with speckle-tracking imaging 
Mild to moderate pneumonia was defined as the patients with laboratory and thorax CT-confirmed interstitial pneumonia in the absence of clinical signs of severe pneumonia. The patients without pneumonia were defined as patients without signs of pneumonia with laboratory or thorax CT scan.

\section{Statistical analysis}

All statistical tests were conducted using the Statistical Package for the Social Sciences 26.0 for Windows (SPSS Inc., Chicago, IL, USA). The Kolmogorov-Smirnov test was used to analyze the normality of the data. Continuous data are expressed as mean \pm standard deviation (SD), and categorical data are expressed as percentages. A Chi-square or Fisher's exact test was used to assess the differences in categorical variables between the groups. A Student's t-test or the Mann-Whitney U test was used to compare unpaired samples as needed. The relationships among the parameters were assessed using Pearson's or Spearman's correlation analysis according to the normality of the data. The primary analysis used ANOVA to compare all reported data for parametric variables, whereas the Kruskal-Wallis test was used for comparison among non-parametric variables between groups. Univariate and multivariate logistic regression analyses were used to identify the independent variables of impaired RVLS. After performing univariate analysis, the stepwise method was used to select significant obtained variables for use in the multivariate logistic regression analysis. The results of the univariate and multivariate regression analyses are presented as odds ratios with $95 \%$ confidence intervals (CIs). Significance was assumed at a two-sided $\mathrm{p}<0.05$.

\section{Results}

\section{Clinical characteristics}

A total of 120, 79 of them recovered from COVID-19 and 41 risk factor-matched controls were included in the present study. At the time of diagnosis of COVID-19, a total of $36(45 \%)$ severely unwell patients required hospitalization. Forty three (55\%) patients recovered at home. The mean age of the home recovery group was $46.7 \pm 12.5$, and the hospital recovery group was $53 \pm 14.1$. There were no statistical differences between home recovery, hospital recovery and control groups in terms of age, gender, body mass index (BMI), heart rate, and preexisting cardiovascular conditions such as stage $1 \mathrm{HTN}$, controlled DM and smoking. Male gender was dominant in all groups. The mean BMI was higher in hospital recovery group while there was no significant difference $(29.2 \pm 4.1$ vs. $27.9 \pm 4.9 ; \mathrm{p}=0.052)$.
Typical pneumonia was diagnosed in 39 patients (49\%) with COVID-19. In hospitalized patients, typical pneumonia was more frequently encountered on CT images compared to treated at home $(75 \%, \mathrm{n}=27$ vs. $28 \%, \mathrm{n}=12 ;[\mathrm{p}<0.001])$. In a subgroup analysis of 39 patients with pneumonia, 11 patients had severe pneumonia, all requiring hospitalization, and 28 patients had mild-moderate pneumonia, of which 16 required hospitalization.

Eleven (31\%) of 36 hospitalized patients had severe pneumonia required high flow nasal cannula (HFNC)/noninvasive ventilation with continuous positive airway pressure $(22 \%$, $\mathrm{n}=8)$ or orotracheal intubation $(8 \%, \mathrm{n}=3)$. Four patients $(11 \%)$ were admitted to the intensive care unit. Nasal oxygen supplementation was required in all patients at hospital. In addition to respiratory support, hospitalized patients received hydrochloroquine $(100 \%, \mathrm{n}=36)$ antiviral (favipiravir) $(42 \%, \mathrm{n}=15)$, azithromycin $(81 \%, \mathrm{n}=29)$, antibiotics (other than azithromycin) $(58 \%, \mathrm{n}=21)$, low molecular weight heparin $(\mathrm{LMWH})(89 \%, \mathrm{n}=32)$, immune modulator (anakinra or tocilizumab) $(42 \%, \mathrm{n}=15)$, and steroid $(14 \%$, $\mathrm{n}=5$ ) therapy in hospital. Five patients who needed steroid therapy were all in the severe pneumonia group.

The patients treated at home received hydrochloroquine $(100 \%, \mathrm{n}=43)$, antiviral (favipiravir) $(2 \%, \mathrm{n}=1)$, azithromycin $(16 \%, \mathrm{n}=7)$, low molecular weight heparin (LMWH) $(33 \%, \mathrm{n}=14)$, immune modulator (anakinra) $(2 \%, \mathrm{n}=1)$, and antibiotics (other than azithromycin) $(28 \%, \mathrm{n}=12)$ therapy. Our study group has patients recovered from the first wave of COVID-19, we can explain why drug use rates were like that. Favipiravir has not been used routinely yet, it was given only to critically ill patients. In our country, the rate of use of favipiravir in both inpatient and outpatient patients is much higher now.

At hospital admission, the mean hsTnT, pro BNP, $\mathrm{CRP}, \mathrm{D}$-dimer, and ferritin values were higher $(\mathrm{p}=0.003$; $\mathrm{p}=0.039 ; \mathrm{p}<0.001 ; \mathrm{p}<0.001 ; \mathrm{p}<0.001 ;$ respectively) and the mean heamoglobin, leukocyte, and lymphocyte values were lower $(p=0.003 ; p=0.014 ; p<0.001$; respectively) in hospitalized patients compared to treated at home.

At follow-up, the mean hs-TnT was higher in hospital recovery group compared to home recovery group $(p=0.021)$, and the mean D-dimer and pro-BNP values were higher in hospital recovery group compared to control group $(\mathrm{p}=0.002 ; \mathrm{p}=0.018$; respectively).

The clinical, demographic features and laboratory findings of home-recovery, hospital-recovery and risk factormatched control groups are presented in Table 1.

\section{Echocardiographic characteristics}

A comparison of the LVEF, LVEDV, LVESV, LVEDD, LV mass index, LAVI, mitral $\mathrm{E}$ velocity and the E/e' ratio of 
Table 1 The clinical, demographic features and laboratory findings of home-recovery, hospital-recovery and risk factor-matched control groups

\begin{tabular}{|c|c|c|c|c|c|}
\hline & $\begin{array}{l}\text { Total patients } \\
(\mathrm{n}=120)\end{array}$ & $\begin{array}{l}\text { Risk factor } \\
\text { matched-controls } \\
(\mathrm{n}=41)\end{array}$ & $\begin{array}{l}\text { Home recovery } \\
(\mathrm{n}=43)\end{array}$ & $\begin{array}{l}\text { Hospital recovery } \\
(\mathrm{n}=36)\end{array}$ & p-value \\
\hline \multicolumn{6}{|c|}{ Clinical characteristics and comorbidities } \\
\hline Age (year) & $49.5 \pm 13.2$ & $49.1 \pm 12.7$ & $46.7 \pm 12.5$ & $53.3 \pm 14.1$ & 0.084 \\
\hline $\begin{array}{l}\text { Gender, n (\%) Male } \\
\text { Female }\end{array}$ & $\begin{array}{l}62(51.7 \%) \\
58(48.3 \%)\end{array}$ & $\begin{array}{l}19(46 \%) \\
22(54 \%)\end{array}$ & $\begin{array}{l}24(56 \%) \\
19(44 \%)\end{array}$ & $\begin{array}{l}19(53 \%) \\
17(47 \%)\end{array}$ & 0.791 \\
\hline $\mathrm{HT}, \mathrm{n}(\%)$ & $36(30 \%)$ & $11(27 \%)$ & $12(28 \%)$ & $13(36 \%)$ & 0.131 \\
\hline DM, n (\%) & $19(16.1 \%)$ & $6(15 \%)$ & $4(10 \%)$ & $9(26 \%)$ & 0.149 \\
\hline Smoker, n (\%) & $35(29 \%)$ & $16(39 \%)$ & $12(28 \%)$ & $7(19 \%)$ & 0.360 \\
\hline $\mathrm{HR}(\mathrm{bpm})$ & $79.3 \pm 12.9$ & $76.5 \pm 7.1$ & $77.9 \pm 14.2$ & $81.9 \pm 12.3$ & 0.187 \\
\hline $\begin{array}{l}\text { BMI }\left(\mathrm{kg} / \mathrm{m}^{2}\right) \\
\text { Pneumonia on CT }\end{array}$ & $\begin{array}{l}28.5 \pm 4.5 \\
39(33 \%)\end{array}$ & $\begin{array}{l}28.7 \pm 3.9 \\
-\end{array}$ & $\begin{array}{l}27.9 \pm 4.9 \\
12(28 \%)\end{array}$ & $\begin{array}{l}29.2 \pm 4.1 \\
27(75 \%)\end{array}$ & $\begin{array}{r}0.052 \\
<0.001^{*}\end{array}$ \\
\hline Follow-up duration (days) & $133 \pm 35$ & - & $130 \pm 34$ & $136 \pm 35$ & 0.448 \\
\hline \multicolumn{6}{|c|}{ Laboratory findings on the day of 2D TTE } \\
\hline $\operatorname{Hgb}(\mathrm{gr} / \mathrm{dl})$ & $13.2(9-17)$ & $13.3(13-17)$ & $13.3(9-16)$ & $13.2(9-16)$ & 0.270 \\
\hline $\operatorname{WBC}\left(10^{3} / \mu \mathrm{l}\right)$ & $6.7(2.4-19.6)$ & $6.9(4.4-13.5)$ & $6.1(3.4-10.8)$ & $6.8(2.4-19.6)$ & 0.229 \\
\hline Lymphocytes $\left(10^{3} / \mu \mathrm{l}\right)$ & $2.2(0.5-6.9)$ & $2.3(1.1-6.9)$ & $2.1(1-4.1)$ & $2.1(0.5-4.1)$ & 0.254 \\
\hline Hs-troponin-T (pg/ml) & $4.6(3-66)$ & $3(3-10)$ & $4.2(3-37)^{\mathrm{c}}$ & $7.4(3-66)^{\mathrm{c}}$ & $0.021^{*}$ \\
\hline Pro-BNP (pg/ml) & $48(4-358)$ & $20.7(4-35)^{b}$ & $57(5-163)$ & $66.8(5-358)^{\mathrm{b}}$ & $0.018^{*}$ \\
\hline CRP (mg/L) & $2.1(0-19)$ & $1.8(1-16)$ & $1.6(0-17)$ & $2.8(0-19)$ & 0.111 \\
\hline D-dimer $(\mu \mathrm{g} / \mathrm{L})$ & $340(180-910)$ & $340(270-500)^{\mathrm{b}}$ & $320(190-910)$ & $360(180-840)^{\mathrm{b}}$ & $0.002 *$ \\
\hline Ferritin (ng/ml) & $54.9(7-510)$ & $60.6(21-97)$ & $96.5(7-486)$ & $51.8(11-510)$ & 0.054 \\
\hline \multicolumn{6}{|c|}{ Laboratory findings at hospital admission } \\
\hline $\mathrm{Hgb}(\mathrm{gr} / \mathrm{dl})$ & $13.2(8.1-16.8)$ & - & $13.7(9.8-16.8)$ & $12.4(8.1-16.1)$ & 0.003 \\
\hline $\operatorname{WBC}\left(10^{3} / \mu \mathrm{l}\right)$ & $6.4(2.8-15.9)$ & - & $5.8(4.4-10.6)$ & $4.9(2.8-15.9)$ & 0.014 \\
\hline Lymphocytes $\left(10^{3} / \mu \mathrm{l}\right)$ & $1.9(0.08-6.9)$ & - & $1.7(0.5-3.5)$ & $0.9(0.08-5.1)$ & $<0.001$ \\
\hline Hs-troponin-T (pg/ml) & $4.2(3-50.9)$ & - & $4.2(3-50.9)$ & $7.6(3-37.4)$ & 0.003 \\
\hline Pro-BNP (pg/ml) & $75.5(4.3-4269)$ & - & $80.9(7.4-619.5)$ & $84.9(8.5-4269)$ & 0.039 \\
\hline $\mathrm{CRP}(\mathrm{mg} / \mathrm{L})$ & $9.6(0.2-230)$ & - & $8.5(0-56)$ & $51.7(3-230)$ & $<0.001$ \\
\hline D-dimer $(\mu \mathrm{g} / \mathrm{L})$ & $543(210-18,550)$ & - & $345(210-920)$ & $1010(320-18,550)$ & $<0.001$ \\
\hline Ferritin (ng/ml) & $96(9-1718)$ & - & $82(14-702)$ & $413(40-1718)$ & $<0.001$ \\
\hline \multicolumn{6}{|l|}{ Treatment } \\
\hline Hydroxychloroquine, n (\%) & $79(65.8 \%)$ & - & $43(100 \%)$ & $36(100 \%)$ & 1.000 \\
\hline Azithromycin, n (\%) & $36(30 \%)$ & - & $7(16.3 \%)$ & $29(80.6 \%)$ & $<0.001$ \\
\hline $\begin{array}{l}\text { Antiviral (Favipiravir), n (\%) } \\
\text { Heparin (LMWH), n (\%) }\end{array}$ & $\begin{array}{l}16(13.3 \%) \\
46(58 \%)\end{array}$ & - & $\begin{array}{l}1(2.3 \%) \\
14(33 \%)\end{array}$ & $\begin{array}{l}15(41.7 \%) \\
32(89 \%)\end{array}$ & $\begin{array}{l}<0.001 \\
<0.001\end{array}$ \\
\hline Steroid, n (\%) & $5(4.2 \%)$ & - & 0 & $5(13.9 \%)$ & 0.012 \\
\hline Immune modulator, n (\%) & $16(13.3 \%)$ & - & $1(2.3 \%)$ & $15(41.7 \%)$ & $<0.001$ \\
\hline Antibiotics, n (\%) & $33(27.5 \%)$ & - & $12(27.9 \%)$ & $21(58.3 \%)$ & 0.006 \\
\hline Hospital stay (days) & $5(2-45)$ & - & 0 & $5(2-45)$ & $<0.001$ \\
\hline ICU admission, n (\%) & $4(3.3 \%)$ & - & $0(0 \%)$ & $4(11.1 \%)$ & 0.041 \\
\hline HFNC/NIMV, n(\%) & $8(6.6 \%)$ & - & $0(0 \%)$ & $8(22.2 \%)$ & 0.011 \\
\hline Orotracheal intubation, $\mathrm{n}(\%)$ & $3(2.5 \%)$ & - & $0(0 \%)$ & $3(8.3 \%)$ & 0.055 \\
\hline
\end{tabular}

$H T$ hypertension, $D M$ diabetes mellitus, $C A D$ coronary artery disease, $C O P D$ chronic obstructive pulmonary disease, $H R$ heart rate, $B M I$ body mass index, $C T$ computed tomography, Pro- $B N P$ pro-brain natriuretic peptid, $H s$-troponin- $T$ high sensitive troponin-T, $H g b$ haemoglobin, $W B C$ white blood cell, $C R P$ C-reactive protein, $L M W H$ low molecular weight heparin, $H F N C$ high flow nasal cannula, NIMV non-invasive mechanic ventilation, $I C U$ intensive care unit

${ }^{\mathrm{a}} \mathrm{p}<0.05$ between control group and home-recovery group

${ }^{\mathrm{b}} \mathrm{p}<0.05$ between control and hospital-recovery group

${ }^{c} \mathrm{p}<0.05$ between home-recovery and hospital-recovery group 


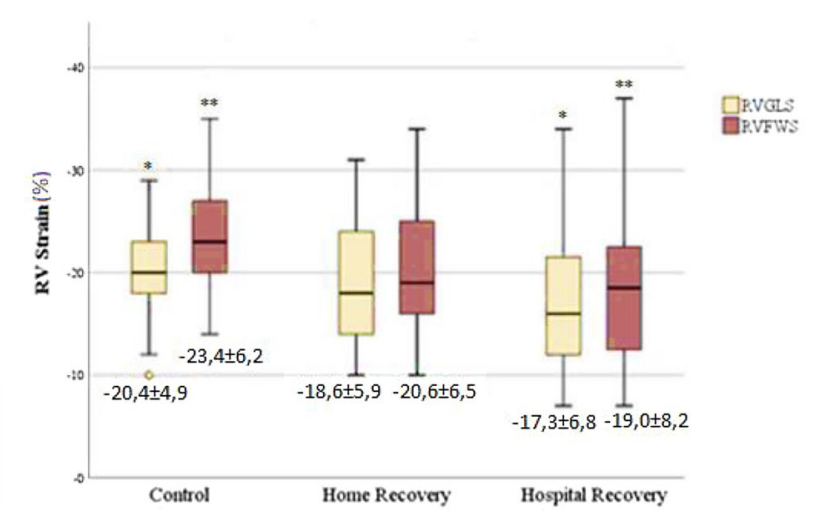

* : a significant difference between hospital recovery and control groups for RV-GLS ( $\mathrm{p}=0,042)$

** : a significant difference between hospital recovery and control groups for RV-FWS $(\mathrm{p}=0,022)$

Fig. 2 The comparison of RV-GLS and RV-FWS between homerecovery, hospital-recovery and control groups. *a significant difference between hospital recovery and control groups for RV-GLS $(\mathrm{p}=0.042) . * *$ a significant difference between hospital recovery and control groups for RV-FWS $(\mathrm{p}=0.022)$ the home-recovery, hospital-recovery and control groups revealed no statistically significant difference.

RV diameter was increased in hospital recovery group compared to controls $(\mathrm{p}=0.036)$, and sPAP was higher in hospital and home recovery groups compared to controls ( $\mathrm{p}<0.001$ for each). RA, TAPSE, FAC, and TDI S' were similar between the groups.

Using 2D STE, RV-GLS and RV-FWS were evaluated in each group. The RV-GLS values of the control, home recovery, and hospital recovery groups were $-20.4 \pm 4.9 \%$, $-18.6 \pm 5.9 \%$, and $-17.3 \pm 6.8 \%$; respectively, indicating a statistically significant difference between hospital recovery and control groups $(p=0.042)$. RV-FWS values of the control, home recovery, and hospital recovery groups were $-23.4 \pm 6.2 \%,-20.6 \pm 6.5 \%$, and $-19.0 \pm 8.2 \%$; respectively, again indicating a statistically significant difference between hospital recovery and control groups $(\mathrm{p}=0.022)$ (Fig. 2).

Echocardiographic parameters of home-recovery, hospital-recovery and control groups are presented in Table 2.
Table 2 Echocardiographic parameters of home-recovery, hospital-recovery and control groups

\begin{tabular}{lccccc}
\hline & $\begin{array}{l}\text { Total patients } \\
(\mathrm{n}=120)\end{array}$ & $\begin{array}{l}\text { Control } \\
(\mathrm{n}=41)\end{array}$ & $\begin{array}{l}\text { Home recovery } \\
(\mathrm{n}=43)\end{array}$ & $\begin{array}{l}\text { Hospital recovery } \\
(\mathrm{n}=36)\end{array}$ & p-value \\
\hline Left heart findings & & & & & \\
LVEDV (ml) & $137.4 \pm 29.9$ & $128.9 \pm 24.6$ & $140.7 \pm 24.5$ & $143.2 \pm 38.8$ & 0.063 \\
LVESV (ml) & $54.5 \pm 16.7$ & $50.7 \pm 12$ & $56.01 \pm 12.2$ & $56.9 \pm 24.2$ & 0.058 \\
LVEDD (mm) & $45.2 \pm 4.5$ & $43.9 \pm 4.1$ & $45.9 \pm 3.9$ & $45.9 \pm 5.5$ & 0.065 \\
LVEF (\%) & $64.6 \pm 5.8$ & $65 \pm 3.9$ & $64.6 \pm 5.5$ & $64 \pm 7.7$ & 0.889 \\
LAVI (ml/m2) & $18.3 \pm 6.3$ & $18.1 \pm 5.9$ & $18.1 \pm 6.2$ & $19.5 \pm 4.9$ & 0.620 \\
E (cm/sn) & $64.2 \pm 16.8$ & $66.7 \pm 16.6$ & $66.4 \pm 16.9$ & $61 \pm 16.4$ & 0.129 \\
E/e' ratio & $8.8 \pm 2.9$ & $8.5 \pm 2.6$ & $8.7 \pm 3.2$ & $9.3 \pm 3.13$ & 0.403 \\
Right heart findings & & & & & \\
RV (mm) & $26.7 \pm 2.6$ & $25.9 \pm 2.7^{\mathrm{b}}$ & $27.1 \pm 3.1$ & $27.1 \pm 1.8^{\mathrm{b}}$ & $0.036^{*}$ \\
RA (mm) & $31.3 \pm 3.2$ & $30.8 \pm 2.8$ & $31.6 \pm 3.7$ & $31.6 \pm 2.7$ & 0.472 \\
RV FAC $(\%)$ & $56.6 \pm 14.1$ & $54.9 \pm 13.1$ & $59.4 \pm 10.3$ & $54.8 \pm 18.6$ & 0.302 \\
RIMP & $0.48 \pm 0.06$ & $0.46 \pm 0.07$ & $0.49 \pm 0.07$ & $0.49 \pm 0.05$ & 0.106 \\
RV GLS $(\%)$ & $-18.9 \pm 6$ & $-20.4 \pm 4.9^{\mathrm{b}}$ & $-18.6 \pm 5.9$ & $-17.3 \pm 6.8^{\mathrm{b}}$ & $0.042^{*}$ \\
RV FWS (\%) & $-21.1 \pm 7.1$ & $-23.4 \pm 6.2^{\mathrm{b}}$ & $-20.6 \pm 6.5$ & $-19 \pm 8.2^{\mathrm{b}}$ & $0.022^{*}$ \\
TAPSE (mm) & $21.9 \pm 3.5$ & $22.9 \pm 3.3$ & $21.4 \pm 3.7$ & $21.5 \pm 3.4$ & 0.092 \\
sPAP (mmHg) & $23.3 \pm 6.2$ & $18.1 \pm 4.9^{\mathrm{a} . \mathrm{b}}$ & $25.1 \pm 4.7^{\mathrm{a}}$ & $27.1 \pm 5.1^{\mathrm{b}}$ & $<0.001^{*}$ \\
TDI S' (cm/s) & $14 \pm 2.6$ & $13.5 \pm 2.2$ & $14.1 \pm 2.8$ & $14.5 \pm 2.7$ & 0.481 \\
\hline
\end{tabular}

$L V$ left ventricular, $L V E D V$ left ventricular end-diastolic volume, $L V E S V$ left ventricular end-systolic volume, $L V E F$ left ventricular ejection fraction, $L V M I$ left ventricular mass index, $L V E D D$ left ventricular end-diastolic diameter, IVS interventricular septum, $L A V I$ left atrial volume index, $E$ early diastolic transmitral flow, $e$ ' early diastolic tissue velocity, $R V$ right ventricular, $R A$ right atrial, $R V F A C$ right ventricular fractional area change, $R I M P$ right ventricular myocardial performance index, $R V G L S$ right ventricular global longitudinal strain, $R V F W S$ right ventricular free wall strain, TAPSE tricuspid annular plane systolic excursion, PASP pulmonary artery systolic pressure, TDI S' tissue Doppler velocity of the basal free lateral wall of the right ventricle. RVGLS and RVFWS values are absolute values

${ }^{\mathrm{a}} \mathrm{p}<0.05$ between control group and home-recovery group

${ }^{b} \mathrm{p}<0.05$ between control and hospital-recovery group

${ }^{c} \mathrm{p}<0.05$ between home-recovery and hospital-recovery group 
Table 3 Right heart findings of patients with severe pneumonia, mild-moderate pneumonia, without pneumonia and control groups

\begin{tabular}{lccccc}
\hline & $\begin{array}{l}\text { Control } \\
(\mathrm{n}=41)\end{array}$ & $\begin{array}{l}\text { Pneumonia }(-) \\
(\mathrm{n}=40)\end{array}$ & $\begin{array}{l}\text { Mild-moderate } \\
\text { Pneumonia } \\
(\mathrm{n}=28)\end{array}$ & $\begin{array}{l}\text { Severe Pneumonia } \\
(\mathrm{n}=11)\end{array}$ & p-value \\
\hline $\mathrm{RV}(\mathrm{mm})$ & $25.9 \pm 2.7$ & $26.7 \pm 2.1$ & $27.6 \pm 3.1$ & $27.3 \pm 1.3$ & 0.081 \\
RA(mm) & $30.8 \pm 2.8$ & $31.5 \pm 3.6$ & $31.8 \pm 3.2$ & $31.6 \pm 2.8$ & 0.630 \\
FAC (\%) & $54.9 \pm 13.2$ & $58.3 \pm 10.4$ & $59.2 \pm 13.9$ & $58.3 \pm 16.4$ & 0.494 \\
RIMP & $0.47 \pm 0.07$ & $0.49 \pm 0.07$ & $0.49 \pm 0.07$ & $0.49 \pm 0.04$ & 0.160 \\
RVGLS (\%) & $-20.4 \pm 4.9^{\mathrm{c}}$ & $-18.9 \pm 6.8$ & $-19.2 \pm 6.7$ & $-15.2 \pm 6.9^{\mathrm{c}}$ & $0.013^{*}$ \\
RVFWS (\%) & $-23.4 \pm 6.2^{\mathrm{c}}$ & $-20.8 \pm 7.7^{\mathrm{e}}$ & $-21.6 \pm 7.6^{\mathrm{f}}$ & $-15.8 \pm 7.6^{\mathrm{c}, \mathrm{f}, \mathrm{f}}$ & $0.001^{*}$ \\
sPAP (mmHg) & $18.1 \pm 4.9^{\mathrm{a}, \mathrm{b}, \mathrm{c}}$ & $25.1 \pm 5.1^{\mathrm{a}, \mathrm{d}, \mathrm{e}}$ & $28.0 \pm 4.9^{\mathrm{b}, \mathrm{d}}$ & $27.1 \pm 2.5^{\mathrm{ce}}$ & $<0.001^{*}$ \\
TAPSE (mm) & $22.9 \pm 3.4$ & $21.9 \pm 4.2$ & $20.7 \pm 2.7$ & $18.6 \pm 2.3$ & 0.071 \\
TDI S' $(\mathrm{cm} / \mathrm{s})$ & $14.0 \pm 2.2$ & $14.0 \pm 3.2$ & $14.0 \pm 2.3$ & $12.5 \pm 2.3$ & 0.139 \\
\hline
\end{tabular}

$R V$ right ventricular, $R A$ right atrial, $R V F A C$ right ventricular fractional area change, $R I M P$ right ventricular myocardial performance index, RVGLS right ventricular global longitudinal strain, $R V F W S$ right ventricular free wall strain, TAPSE tricuspid annular plane systolic excursion, PASP pulmonary artery systolic pressure, TDI S' tissue Doppler velocity of the basal free lateral wall of the right ventricle. RVGLS and RVFWS values are absolute values

${ }^{a} p<0.05$ between control group and pneumonia ( - ) group
${ }^{b} p<0.05$ between control and mild-moderate pneumonia group
${ }^{c} p<0.05$ between control and severe pneumonia group
${ }^{d} p<0.05$ between pneumonia ( - ) and mild-moderate pneumonia group
${ }^{e} p<0.05$ between pneumonia ( - ) and severe pneumonia group
${ }^{\mathrm{f}} \mathrm{p}<0.05$ between mild-moderate pneumonia and severe pneumonia group
In a subgroup analysis, right heart findings of 11 patients with severe pneumonia were compared to 28 patients with mild-moderate pneumonia and 40 patients without pneumonia, and control groups (Table 3). A significant difference was found between severe pneumonia and control group according to RV-GLS $(-15.2 \pm 6.9$ vs. $-20.4 \pm 4$, respectively; $\mathrm{p}=0.013)$. Moreover, RV-FWS was impaired in severe pneumonia group compared to mild-moderate pneumonia, without pneumonia and control groups $(-15.8 \pm 7.6 ;-21.6 \pm 7.6 ;-20.8 \pm 7.7$; $-23.4 \pm 6.2$, respectively, $\mathrm{p}=0.001$ for each) (Fig. 3). sPAP was higher in mild-moderate pneumonia and severe pneumonia groups compared to without pneumonia group and in all groups compared to control group $(\mathrm{p}<0.001$ for each).

The relationship between RV-GLS, RV-FWS and age, hs-TnT, D-dimer, CRP, Hgb, ferritin, pro-BNP levels were evaluated via Spearman's or Pearson's correlation analyses. A statistically significant correlation was detected between serum CRP level at hospital admission and both RV-GLS and RV-FWS $(r=0.285, p=0.006 ; r=0.294, p=0.004$, respectively) (Fig. 4). Again age was significantly corralated with RV-GLS and RV-FWS $(r=0.244, p=0.008 ; r=0.176$, $\mathrm{p}=0.054$, respectively) (Table 4 ). There was no correlation between hs-TnT and pro-BNP levels, which are cardiac injury markers, and D-dimer and RVLS (Tables 4, 5).

The parameters affecting impaired RV-FWS $(>-18)$ were evaluated by using logistic regression analysis with

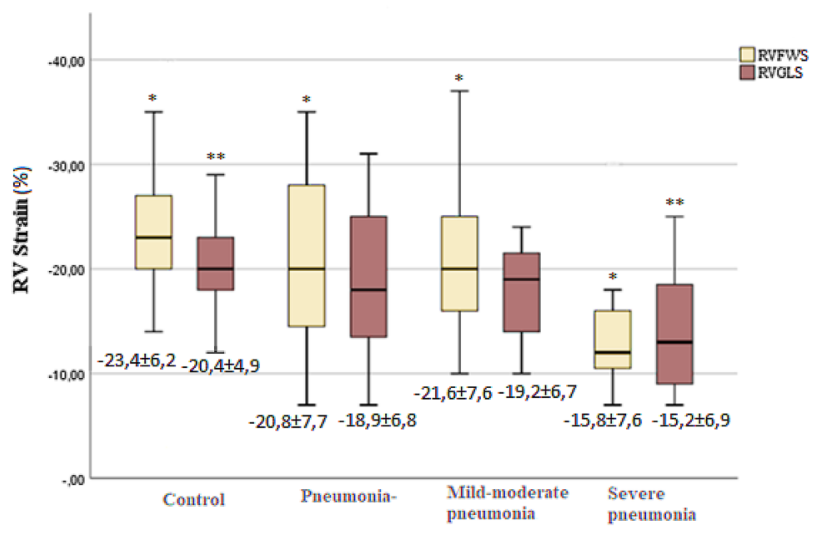

*: RV-FWS was impaired in severe pneumonia group compared to mild-moderate pneumonia, without pneumonia and control groups ( $\mathrm{p}=0,001$ for each).

$* *$ : a significant difference between severe pneumonia and control group for RV-GLS $(\mathrm{p}=0,013)$.

Fig. 3 The comparison of RV-GLS and RV-FWS between patients without pneumonia, mild-moderate pneumonia, severe pneumonia and control groups (Data are presented as mean $\pm \mathrm{SD}$ ). *RV-FWS was impaired in severe pneumonia group compared to mild-moderate pneumonia, without pneumonia and control groups $(\mathrm{p}=0.001$ for each). **a significant difference between severe pneumonia and control group for RV-GLS $(\mathrm{p}=0.013)$

univariate and multivariate analysis. Age (OR 0.948, $\mathrm{p}=0.010)$, male gender (OR $0.289, \mathrm{p}=0.009)$, pneumonia on CT (OR $0.019, \mathrm{p}=0.004)$, and need of steroid in treatment (OR 17.424, $\mathrm{p}=0.038$ ) were thus determined to be independent predictors of impaired RV-FWS (Table 6). 
Fig. 4 a Correlation of RV-GLS with serum CRP level at hospital admission. b Correlation of RV-FWS with serum CRP level at hospital admission
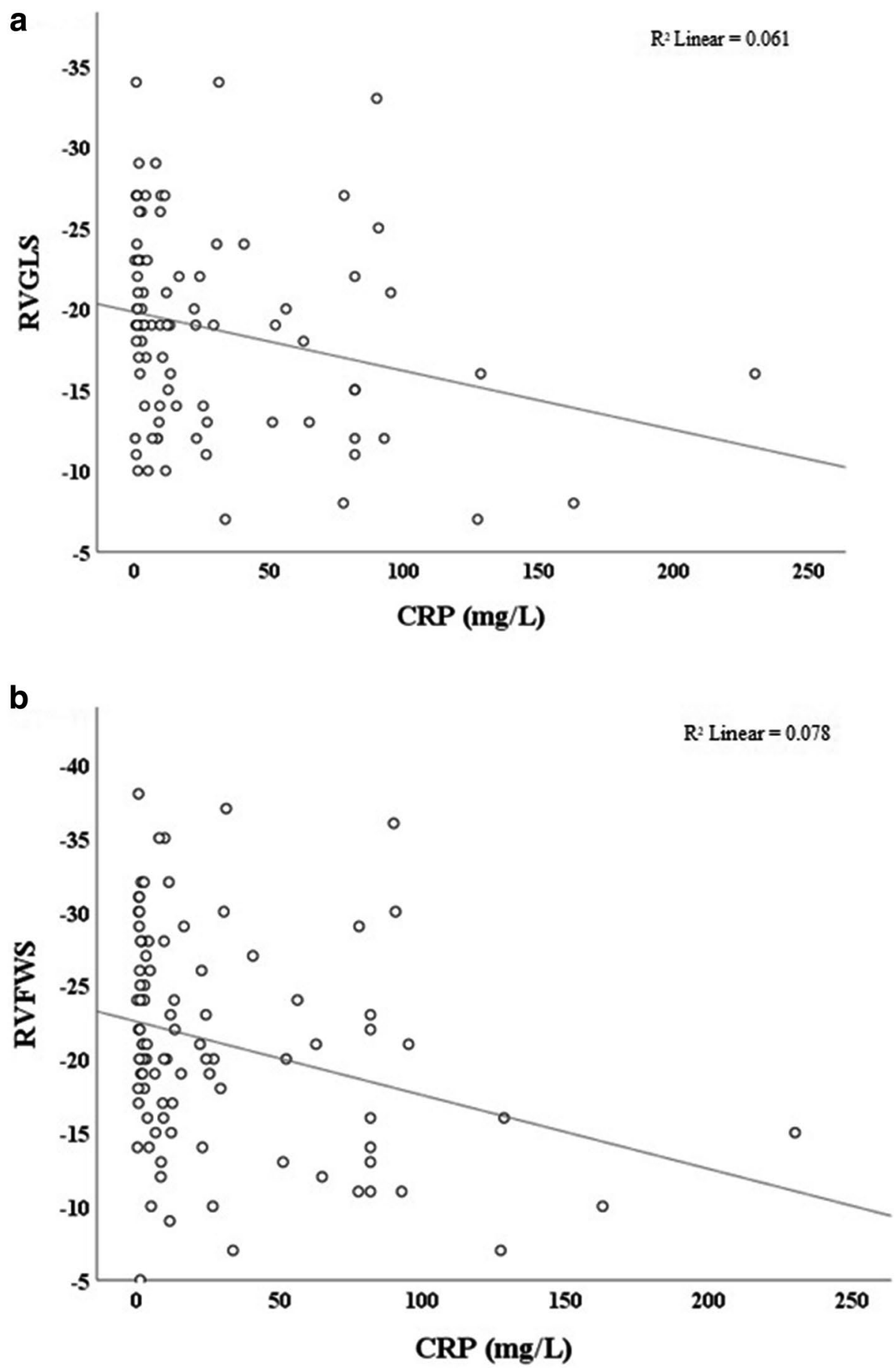

Table 4 Correlation of RV-GLS and RV-FWS with age and laboratory parameters at hospital admission

\begin{tabular}{llrllllll}
\hline & Spearman & RVFWS & Age & Hs-troponin-T & D-dimer & CRP & Ferritin & Pro-BNP \\
\hline RVGLS (\%) & $\mathrm{r}$ & 0.912 & 0.244 & 0.119 & 0.125 & 0.285 & 0.206 & 0.105 \\
& $\mathrm{p}$ & $<0.001^{*}$ & $\mathbf{0 . 0 0 8}$ & 0.326 & 0.333 & $\mathbf{0 . 0 0 6}$ & 0.067 & 0.403 \\
RVFWS (\%) & $\mathrm{r}$ & & 0.176 & 0.060 & 0.165 & 0.294 & 0.203 & 0.081 \\
& $\mathrm{p}$ & $\mathbf{0 . 0 5 4}$ & 0.616 & 0.195 & $\mathbf{0 . 0 0 4}$ & 0.068 & 0.517 \\
\hline
\end{tabular}

$R V G L S$ right ventricular global longitudinal strain, $R V F W S$ right ventricular free wall strain, $h s$-troponin- $T$ high sensitive troponin-T, $C R P$ C-reactive protein, pro-BNP pro-brain natriuretic peptid 
Table 5 Correlation of RV-GLS and RV-FWS with laboratory parameters at follow-up

\begin{tabular}{llclllll}
\hline & Spearman & RVFWS & Hs-troponin-T & D-dimer & CRP & Ferritin & Pro-BNP \\
\hline RVGLS (\%) & $\mathrm{r}$ & 0.912 & 0.015 & 0.118 & 0.132 & 0.126 & 0.085 \\
& $\mathrm{p}$ & $<0.001 *$ & 0.884 & 0.214 & 0.162 & 0.212 & 0.434 \\
RVFWS (\%) & $\mathrm{r}$ & & 0.008 & 0.130 & 0.034 & 0.116 & 0.052 \\
& $\mathrm{p}$ & & 0.936 & 0.169 & 0.720 & 0.246 & 0.631 \\
\hline
\end{tabular}

Table 6 Multivariate logistic regression analysis of the risk factors associated with impaired RV-FWS

\begin{tabular}{lrll}
\hline Variable & \multicolumn{1}{l}{ OR } & $95 \%$ Confidence interval & p-value \\
\hline Age & 0.948 & $0.910-0.987$ & $\mathbf{0 . 0 1 0} *$ \\
Gender & 0.289 & $0.114-0.733$ & $\mathbf{0 . 0 0 9 *}$ \\
Pneumonia on CT & 0.019 & $0.001-0.290$ & $\mathbf{0 . 0 0 4} *$ \\
$\begin{array}{l}\text { Need of steroid in treat- } \\
\text { ment }\end{array}$ & 17.424 & $1.172-259.093$ & $\mathbf{0 . 0 3 8} *$ \\
\hline
\end{tabular}

$R V F W S$ right ventricular free wall strain, $C T$ computed tomography

\section{Discussion}

This study was done in survivors of COVID-19 with a median $133 \pm 35$ days after recovery and shows that; in patients recovered from hospital, RV diameter was increased and RV-GLS and RV-FWS were impaired compared to control group, and sPAP was higher in hospital and home recovery groups compared to controls, while left heart findings were similar between groups. In subgroup analysis, in patients with severe pneumonia, RV-GLS was impaired compared to control group and RV-FWS was impaired compared to mild-moderate pneumonia, without pneumonia and control groups. sPAP was higher in severe pneumonia and mild-moderate pneumonia groups compared to without pneumonia and control groups. Age, male gender, pneumonia on CT, and need of steroid in treatment were identifed as independent predictors of impaired RVLS through multivariate analysis. A relationship was detected between CRP level at hospital admission and both RV-GLS and RV-FWS. There was no correlation between cardiac injury markers, D-dimer and RVLS. These findings support the right-sided cardiac involvement and the impairment of subclinical RV systolic function by 2D STE in relation to the severity of pneumonia in hospitalized COVID-19 patients and persistence after recovery.

Pulmonary involvement often occurs in COVID19 , so investigators evaluated RV and showed both RV enlargement and dysfunction in hospitalized patients with COVID-19. Again, RV involvement was associated with a poor prognosis in these patients [17-19].

In a multi-center study, Kim et al. evaluated 510 hospitalized patients with COVID-19. RV dilatation was found in $35 \%$, and dysfunction in $15 \%$ of the patients with echocardiographic examination. Both RV dilatation and dysfunction were associated with prognosis, independent of cardiac injury biomarkers, and predicted mortality during hospitalization [17]. Again Mahmoud et al. evaluated 74 hospitalized patients with COVID-19 pneumonia. LV function was normal in $89 \%$ of patients. However, $41 \%$ of patients had RV dilatation and $27 \%$ had RV dysfunction. $\mathrm{RV}$ involvement was associated with increased D-dimer and CRP levels [18]. Considering that the increased CRP level represents the severity of the disease and pneumonia, its relation with RV involvement is reasonable. Supporting this in our study, increased CRP level at hospital admission was associated with impairment in both RV-GLS and RV-FWS after recovery. Inflammatory laboratory parameters rather than cardiac injury markers seem to be predictors of cardiac involvement.

The possible mechanism of RV dilation and dysfunction is likely multifactorial. Thrombotic events, hypoxic vasoconstriction, direct viral damage and proinflammatory cytokines, and most probably increased afterload and overload, are some of these mechanisms [5, 6, 20,21].

Since RV involvement was detected during hospitalization in previous studies, we aimed to investigate whether it persists after recovery. Whether the RV involvement continues in patients after recovery may provide information about prognosis. To avoid acute effects of the infection on cardiac functions, echocardiographic examinations were performed at least 3 months after recovery. In our study, TAPSE, FAC, and TDI S' values were similar between the groups. All patients had normal global RV systolic function. Conventional echocardiography parameters may be insufficient to assess RV function [22]. 2D-STE objectively measures myocardial deformation globally and regionally $[22,23]$. So we evaluated the RVLS with 2D-STE to investigate the subclinic systolic dysfunction. There are few studies evaluating cardiac involvement after recovery from COVID-19.

Catena et al. evaluated 105 patients with COVID-19 who were discharged from the hospital after a median of 41 days. According to conventional echocardiographic parameters, there was no evidence of persistent cardiac dysfunction, even in patients with previously increased troponin. Differences from our study were shorter followup time, no control group and no assessment according 
to the severity of pneumonia and infection. Moreover, no evaluation was made with 2D-STE [24].

In a cardiac magnetic resonance (CMR) study of 100 patients recovered from COVID-19, non-specific markers associated with inflammation was detected in 78 patients. Active lymphocytic inflammation without any evidence of viral genome was demonstrated with endomyocardial biopsy in three patients whom had severe abnormalities [25]. In an another CMR study, Huang et al. evaluated 28 patients recovered from COVID-19 pneumonia. Fifteen patients $(58 \%)$ had abnormal CMR findings including myocardial edema, and LGE. Decreased RV systolic function parameters were detected in patients with abnormal CMR findings however there was no significant difference of LV function compared to healthy controls [26]. CMR is the gold standard imaging modality that assesses ventricular functions. These studies demonstrated that RV is the main target in COVID-19 as we mentioned. Thus, the follow-up of these patients can be done by 2D-STE because of its easy applicability according to CMR in daily practice.

In a multicenter study, pathologists evaluated the cardiac tissue from autopsies of 21 people who died from COVID-19. Three of the cases had lymphocytic myocarditis, 18 had increased interstitial macrophage infiltration, four had acute myocyte injury in the RV. There was no molecular evidence to suggest the presence of the virus. High myocardial macrophage levels were thought to be associated with the elevated proinflammatory cytokines. Acute myocyte injury in RV, detected in $25 \%$ of cases, was attributed to increased strain/afterload [27]. Both CMR and pathology studies observed no virus-specific findings, detected changes consistent with the severity of inflammation in the myocardium. Thereby, the conclusion that the clinical severity of COVID-19 is associated with ongoing or persistent RV dysfunction determined by the 2D-STE we demonstrated is supported. This may not be attributed to COVID-19 as it could be a general outcome that would be expected in patients with similarly severe clinical conditions. Whether our findings and RV involvement are reversible and they affect the long-term prognosis of these patients should be investigated with a longer follow-up study.

Our study has limitations. The sample size was relatively small because the data was derived from a single center. Based upon this being a voluntary follow-up clinic, there may be a selection bias supporting the evaluation of individuals seeking ongoing medical care. Also there was no echocardiographic data of patients prior to COVID19. There were significant differences in drug treatments for COVID-19 in hospital and at home. And finally, the follow-up duration was relatively short.

\section{Conclusion}

We have determined the subclinical impairment of RV function with 2D STE in hospitalized patients in relation to the severity of pneumonia after recovery. In conclusion, right-sided involvement is more likely in patients recovered from COVID-19 pneumonia. These findings may reflect the effects of critical illness, not specific to the virus. Echocardiographers should pay close attention to the early diagnosis of RV dysfunction. The prognosis and long-term cardiovascular conditions of these patients need to be investigated with further studies.

Acknowledgements The authors thank all doctors, nurses, and other health providers at Istanbul Faculty of Medicine who were involved in taking care of COVID-19 patients.

Author contributions All authors contributed to (1) substantial contributions to conception and design, or acquisition of data, or analysis and interpretation of data, (2) drafting the article or revising it critically for important intellectual content, and (3) final approval of the version to be published.

Funding This research did not receive any specific grant from funding agencies in the public, commercial, or not-for-profit sectors.I

\section{Declarations}

Conflict of interest The author(s) declared no potential conflicts of interest with respect to the research, authorship, and/or publication of this article.

\section{References}

1. Zhu N, Zhang D, Wang W, Li X, Yang B, Song J, Zhao X, Huang B, Shi W, Lu R, Niu P, Zhan F, Ma X, Wang D, Xu W, Wu G, Gao GF, Tan W (2020) A novel coronavirus from patients with pneumonia in China 2019. N Engl J Med 382:727-733

2. Shi S, Qin M, Shen B, Cai Y, Liu T, Yang F, Gong W, Liu X, Liang J, Zhao Q, Huang H, Yang B, Huang C (2020) Association of cardiac injury with mortality in hospitalized patients with COVID-19 in Wuhan, China. JAMA Cardiol 5(7):802-810

3. Guo T, Fan Y, Chen M, Wu X, Zhang L, He T et al (2020) Cardiovascular implications of fatal outcomes of patients with coronavirus disease 2019 (COVID-19). JAMA Cardiol 5(7):811-818

4. Wu Z, McGoogan JM (2020) Characteristics of and important lessons from the coronavirus disease (COVID-19) outbreak in China: summary of a report of 72314 cases from the Chinese center for disease control and prevention. JAMA 323:1239-1242

5. Repessé X, Charron C, Vieillard-Baron A (2012) Right ventricular failure in acute lung injury and acute respiratory distress syndrome. Minerva Anestesiol 78:941-948

6. Osman D, Monnet X, Castelain V (2009) Incidence and prognostic value of right ventricular failure in acute respiratory distress syndrome. Intensive Care Med 35:69-76

7. Yang X, Yu Y, Xu J, Shu H, Xia J, Liu H et al (2020) Clinical course and outcomes of critically ill patients with SARS-CoV-2 
pneumonia in Wuhan, China: a single-centered, retrospective, observational study. Lancet Respir Med 8(5):475-481

8. Carluccio E, Biagioli P, Alunni G, Murrone A, Zuchi C, Coiro S et al (2018) Prognostic value of right ventricular dysfunction in heart failure with reduced ejection fraction: superiority of longitudinal strain over tricuspid annular plane systolic excursion. Circ Cardiovasc Imaging 11(1):e006894

9. Li Y, Li H, Zhu S, Xie Y, Wang B, He L et al (2020) Prognostic value of right ventricular longitudinal strain in patients with COVID-19. JACC Cardiovasc Imaging 13(11):2287-2299

10. Baycan OF, Barman HA, Atici A, Tatlisu A, Bolen F, Ergen P et al (2020) Evaluation of biventricular function in patients with COVID-19 using speckle tracking echocardiography. Int J Cardiovasc Imaging 15:1-10

11. Mitchell C, Rahko PS, Blauwet LA, Canaday B, Finstuen JA, Foster MC et al (2019) Guidelines for performing a comprehensive transthoracic echocardiographic examination in adults: recommendations from the American society of echocardiography. $\mathrm{J}$ Am Soc Echocardiogr 32:1-64

12. Lang RM, Badano LP, MorAvi V, Aflalo J, Armstrong A, Ernande L et al (2015) Recommendations for cardiac chamber quantification by echocardiography in adults: an update from the American society of echocardiography and the European association of cardiovascular 1maging. Eur Heart J Cardiovasc Imaging 16(3):233-271

13. Rudski LG, Lai WW, Afilalo J et al (2010) Guidelines for the echocardiographic assessment of the right heart in adults: a report from the American society of echocardiography endorsed by the European association of echocardiography and the canadian society of echocardiography. J Am Soc Echocardiogr 23:685-713

14. Longobardo L, Suma V, Jain R, Carerj S, Zito C, Zwicke DL, Khandheria BK (2017) Role of two-dimensional speckle-tracking echocardiography strain in the assessment of right ventricular systolic function and comparison with conventional parameters. J Am Soc Echocardiogr 30(10):937-946.e6

15. Mor-Avi V, Lang RM, Badano LP et al (2011) Current and evolving echocardiographic techniques for the quantitative evaluation of cardiac mechanics: ASE/ EAE consensus statement on methodology and indications endorsed by the Japanese society of echocardiography. Eur J Echocardiogr 12:167-205

16. McFee DRB (2020) COVID-19 medical management including world health organization (WHO) suggested management strategies. Dis Mon 66(9): 101068

17. Kim J, Volodarskiy A, Sultana R et al (2020) Prognostic utility of right ventricular remodeling over conventional risk stratification in patients with COVID-19. J Am Coll Cardiol 76(17):1965-1977. https://doi.org/10.1016/j.jacc.2020.08.066
18. Mahmoud-Elsayed HM, Moody WE, Bradlow WM, Khan-Kheil AM, Senior J, Hudsmith LE, Steeds RP (2020) Echocardiographic findings in patients with COVID-19 pneumonia. Can J Cardiol 36(8):1203-1207

19. Argulian E, Sud K, Vogel B, et al. (2020) Right ventricular dilation in hospitalized patients with COVID-19 Infection. J Am Coll Cardiol Img 13(11):2459-2461

20. Clerkin KJ, Fried JA, Raikhelkar J (2020) Coronavirus disease 2019 (COVID-19) and cardiovascular disease. Circulation 141:1648-1655

21. Lindner D, Fitzek A, Brauninger H, Aleshcheva G, Edler C, Meissner K et al (2020) Association of cardiac infection with SARS-CoV-2 in confirmed COVID-19 autopsy cases. JAMA Cardiol. https://doi.org/10.1001/jamacardio.2020.3551

22. Blessberger $\mathrm{H}$, Binder $\mathrm{T}$ (2010) Non-invasive imaging: two dimensional speckle tracking echocardiography: basic principles. Heart 96:716-722

23. Lu KJ, Chen JX, Profitis K, Kearney LG, DeSilva D, Smith G et al (2015) Right ventricular global longitudinal strain is an independent predictor of right ventricular function: a multimodality study of cardiac magnetic resonance imaging, real time three-dimensional echocardiography and speckle tracking echocardiography. Echocardiography 32(6):966-974

24. Catena C, Colussi G, Bulfone L, Da Porto A, Tascini C, Sechi LA (2020) Echocardiographic comparison of COVID-19 patients with or without prior biochemical evidence of cardiac injury after recovery. J Am Soc Echocardiogr S0894-7317(20):30666

25. Puntmann VO, Carerj ML, Wieters I, et al. (2020) Outcomes of cardiovascular magnetic resonance imaging in patients recently recovered from coronavirus disease 2019 (COVID-19). JAMA Cardiol. 5(11):1265

26. Huang L, Zhao P, Tang D, Zhu T, Han R, Zhan C et al (2020) Cardiac involvement in patients recovered from COVID-2019 identified using magnetic resonance imaging. JACC Cardiovasc Imaging 13(11):2330-2339

27. Basso C, Leone O, Rizzo S, De Gaspari M, van der Wal AC, Aubry MC et al (2020) Pathological features of COVID-19-associated myocardial injury: a multicentre cardiovascular pathology study. Eur Heart J 41(39):3827-3835

Publisher's Note Springer Nature remains neutral with regard to jurisdictional claims in published maps and institutional affiliations. 